\title{
Sleep disorders and suicidal ideation in patients with depressive disorder
}

\author{
Sarah Laxhmi Chellappa *, John Fontenele Araújo \\ Post-Graduate Program in Health Sciences, Center of Health Sciences, Federal University of Rio Grande Do Norte, \\ Av. Gal Gustavo Cordeiro de Farias, s/n, CEP 59010-180, Natal, RN, Brazil
}

Received 4 January 2006; received in revised form 6 March 2006; accepted 5 May 2006

\begin{abstract}
An intrinsic association between suicidal ideation and sleep disorders in patients with depressive disorder has been observed in recent studies. This study was conducted in order to examine the relationship between suicidal ideation and sleep disorders, such as insomnia and excessive sleepiness, in outpatients with major depressive disorder. Seventy patients with diagnoses of major depressive disorder were interviewed and assessed with the Sleep Habits Questionnaire and the Beck Scale for Suicidal Ideation (SSI). Data analyses were performed through descriptive analysis, Students $t$-test, Chi-square test and logistic regression model, with a statistical significance of 5\%. In this study, depressed patients had high SSI scores $(6.12 \pm 2.67)$, particularly for active suicidal ideation $(1.61 \pm 0.39)$ and specific plans for suicide components $(1.51 \pm 0.40)$. Depressed patients with insomnia had significantly higher SSI scores $(7.39 \pm 2.84)$, in relation to patients with excessive sleepiness $(3.68 \pm 1.73)$. Furthermore it was observed that insomniac patients had significantly higher scores on the following components: active suicide ideation, specific plans for suicide and previous suicide attempts. The results of multivariate analysis showed that only insomnia had a significant association with suicidal ideation. Thus, sleep disturbances, particularly insomnia, should be considered in the assessment of suicidal risk in outpatients with depressive disorder.
\end{abstract}

(C) 2006 Elsevier Ireland Ltd. All rights reserved.

Keywords: Insomnia; Sleep disorder; Major depressive disorder; Suicide; Epidemiology

\section{Introduction}

Sleep disorders are frequently associated with a wide range of psychiatrics illnesses and are regarded as a characteristic feature of depressive disorder. Depressed patients often report inadequate or non-restorative sleep, as well as difficulty in falling asleep, frequent nocturnal and early morning awakening, decreased total

\footnotetext{
* Corresponding author. Tel./fax: +55 8432154220 .

E-mail address: sarahlc@ig.com.br (S.L. Chellappa).
}

sleep and disturbing dreams (Ohayon, 2000; ICSD, 2001).

Suicidal patients habitually report problems with their sleep. Although sleep-related complaints and electroencephalographic changes are generally encountered in psychiatric disorders, sleep complaints, such as insomnia, hypersomnia and nightmares, are more common in suicidal patients (Agargun and Cartwright, 2003; Bernert et al., 2005). Dream variables collected during rapid eye movement (REM) interruptions and REM sleep abnormalities have also been related to suicidal tendencies in depressed patients (Agargun et al., 
1998; Agargun and Cartwright, 2003), as well and the frequency of nightmares has been directly related to suicide risk (Tankensen et al., 2001). Sleep studies have reported various polysomnographic findings including increased REM time and activity in suicidal patients with depression. One mechanism that may be responsible for the association between suicide and sleep is low function in patients who attempted and/or completed suicide (Singareddy and Balon, 2001).

Previous epidemiological studies have indicated that sleep disorders, particularly insomnia, can be associated with suicidal risk (Duggan et al., 1992; Agargun et al., 1997a). Severe insomnia at the time of depression diagnosis has been recognized as one of the few clinical predictors of completed suicide in the first year of follow-up (Fawcett et al., 1990). Moreover, suicidal thinking during depression is frequent in patients with insomnia in comparison to depressed patients without insomnia (Sabo et al., 1991). Recent studies have demonstrated a correlation between suicidal ideation and suicide attempts that can result in an elevated suicide risk at some point in the future (Bertolote and Fleishmann, 2002; Schmidtke et al., 2004). Therefore, the importance of the clinical evaluation of sleep disorders in patients with major depressive disorder is due to its link to severe depression, suicidal ideation and suicidal risk.

\section{Methods}

\subsection{Design and setting}

The study design was descriptive, quantitative and cross-sectional with a sample composed of 70 outpatients. The study was conducted in the Onofre Lopes University Hospital Psychiatry Unit, a public tertiary general hospital in Natal, Rio Grande do Norte, Brazil. Data collection was done during the period of April to July 2005.

\subsection{Participants}

The selection of patients was according to the inclusion criteria of age between 18 and 65 years old and patients with diagnoses of major depressive disorder. This diagnosis was provided through clinical interview using the Diagnostic and Statistical Manual of Mental Disorders criteria (DSM-IV, 1994) for major depressive disorder by five psychiatrics responsible for the clinical evaluation of the patients. After the interviews, medical records were reviewed to assess the physician's recognition of major depressive disorder. All patients consecutively admitted in the psychiatric outpatient unit that met the inclusion criteria of this study were interviewed. The exclusion criteria were as follows: psychoactive substance use, post-traumatic stress disorder, post-puerperal depression, bipolar disorder, and epilepsy.

\subsection{Procedures}

The interviews were conducted by one of the study investigators who had been trained to apply the methodological instruments (Sleep Habits Questionnaire and Beck Scale for Suicide Ideation). All subjects gave written informed consent to participate in this study. The project was approved by the Ethics Committee of the Federal University of Rio Grande do Norte, Natal, Brazil.

Data concerning the duration of major depressive disorder in months were obtained from medical records. Suicidal ideation was assessed with the Beck Scale for Suicidal Ideation (SSI), a clinician rating scale with a semi-structured interview format. This questionnaire is a well-established instrument for assessing suicidal ideation (Beck et al., 1979). It consists of 19 items that evaluate the following dimensions of suicide ideation: active suicide desire, specific plans for suicide, passive suicide desire, and previous suicide attempts. Each item is rated on a scale from 0 to 2 , and these items are grouped into four components: active suicide ideation, specific plans for suicide, passive suicide ideation and previous suicide attempts. Higher scores are related to severe suicidal ideation. A total SSI score equivalent to 6 or more is considered as a cutoff threshold for clinically significant suicidal ideation. In this study, the validated version in Brazil was used to assess suicidal ideation (Cunha, 2001).

Sleep assessment was conducted using the Sleep Habits questionnaire (SHQ) and the International Classification of Sleep Disorders diagnostic criteria for sleep disorders due to mood disorders (ICSD, 2001). The SHQ consists of a standard and validated self-evaluating protocol with 32 questions, out of which 10 items are related to the patients' health and use of stimulants, and 22 items concern sleep disturbances. The SHQ is utilized to assess sleep disorder complaints, notably insomnia and excessive sleepiness, and to classify patients as having insomnia, excessive sleepiness or none of these sleep disturbances. It does not provide an adequate assessment of other sleep disorders, e.g., the obstructive sleep apnea or hypopnea syndrome, nightmares, and restless leg syndrome/periodic limb movements in sleep. Insomnia was defined as difficulty in initiating sleep, disrupted sleep, early morning awakening and insufficient amount of sleep (decreased total duration of 
Table 1

Comparison of SSI components between patients with insomnia and excessive sleepiness with depressive disorder

\begin{tabular}{llll}
\hline SSI $^{\mathrm{a}}$ components & \multicolumn{2}{l}{ Patients $(n=70)$} & \\
\cline { 2 - 3 } & $\begin{array}{l}\text { Insomnia } \\
(n=49)\end{array}$ & $\begin{array}{l}\mathrm{ES}^{\mathrm{c}} \\
(n=21)\end{array}$ \\
\hline
\end{tabular}

$\begin{array}{llllll}\text { Active suicidal ideation } & 1.51 \pm 0.32 & 0.62 \pm 0.30 & 3.84 & 20 & 0.01 \text { * }\end{array}$

Specific plans for suicide $1.48 \pm 0.41 \quad 0.72 \pm 0.35 \quad 5.58 \quad 20 \quad 0.02 *$

$\begin{array}{llllll}\text { Passive suicidal ideation } & 1.22 \pm 0.55 & 1.01 \pm 0.48 & 3.84 & 20 & 0.02\end{array}$

$\begin{array}{lllllll}\text { Previous suicide attempts } & 1.14 \pm 0.20 & 0.39 \pm 0.15 & 2.93 & 20 & 0.18\end{array}$

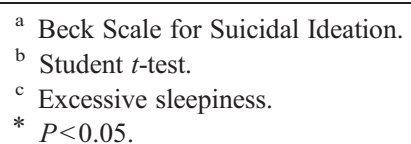

sleep) at least three times a week during the last 30 days, with distress in daily living (Andrade et al., 1992). To be classified by the SHQ as having insomnia, the previously referred insomnia complaints should be present (all the answers to the items regarding insomnia should be affirmative). Excessive sleepiness was defined as an increased propensity to fall asleep and a subjective compulsion to sleep, as well as a tendency to take involuntary naps when sleep is not desired during the last 30 days (Andrade et al., 1992). Moreover, sleepiness was described as sleep episodes that were present on a daily basis and that occurred during the patients' daily activities, such as working. To be classified as having excessive sleepiness by the SHQ, the previously mentioned sleepiness complaints should be present (all the answers to the items regarding excessive sleepiness should be affirmative). Patients that fulfilled neither the criteria established for insomnia nor those for excessive sleepiness were classified as not presenting these sleep disturbances.

The ICSD diagnostic criteria for sleep disorders due to mood disorders consist of the following: A. The patient has a complaint of insomnia or excessive sleepiness; B. The complaint is temporally associated with a diagnosis of mood disorder; $\mathrm{C}$. The complaint is expected to remit if the mood disorder resolves; D. Polysomnographic monitoring demonstrates either a shortened REM sleep latency or an increased REM density or reduced delta sleep or an increased sleep latency, reduced sleep efficiency, and increased number and duration of awakenings; E. The patient does not have any medical or other mental disorder that can account for the sleep disturbance; F. The sleep disturbance does not meet the diagnostic criteria for other sleep disorders that produce insomnia or excessive sleepiness. To qualify for the ICSD diagnosis, the minimal criteria required are A plus B, the standard adopted in this study.
The reliability of the ICSD diagnostic assessment and the SHQ classification of insomnia or excessive sleepiness was indicated by Kappa coefficient $(\kappa=0.76)$. This value is consider to represent excellent interrater agreement (Landis and Koch, 1977).

\subsection{Data analysis}

The following tests were used in the statistical analysis: 1. Descriptive analysis (mean and standard deviation) of the socio-demographic variables and of the scores obtained from the SSI; 2. Student $t$-test for independent samples to assess the SSI global and component scores (mean and standard deviation) between patients with insomnia and excessive sleepiness; 3. Chisquare test to analyze the association of SSI scores with different age groups (age 18-28; 29-39; 40-50, 51-61, and above 61 years) and with the SHQ items regarding insomnia and excessive sleepiness; 4 . Bivariate analysis to study the association between suicide ideation and co-variables of interest, using odds ratios and their respective confidence intervals as estimates of association. Co-variables showing $P$-values below 0.20 were selected and tested using a multiple logistic model, which was included in the final model variables with $P$-values below 0.05 . Logistic regression models were used to study the multivariate relationships of covariables with suicidal ideation. This analysis was adjusted to the potentially confounding factor of medication (antidepressant and hypnotic) intake. Results were considered statistically significant at $P<0.05$.

Table 2

Total number of patients under antidepressant and hypnotic medication in both groups of the study sample and types of medication used

\begin{tabular}{lll}
\hline Variables & \multicolumn{2}{l}{ Patients $(n=70)$} \\
\cline { 2 - 3 } & $n$ & Frequency $(\%)$ \\
\hline Total number of patients under medication & 27 & 38.4 \\
Patients with insomnia $(n=49)$ & 19 & 27 \\
Types of medication & & \\
$\quad$ Benzodiazepines & 7 & 10 \\
Amitriptyline & 3 & 4.3 \\
Nortriptyline & 2 & 2.8 \\
Sertraline & 2 & 2.8 \\
Fluoxetine & 2 & 2.8 \\
$\quad$ Did not refer & 3 & 4.3 \\
Patients with excessive sleepiness $(n=21)$ & 8 & 11.4 \\
Types of medication & & \\
Benzodiazepines & 3 & 4.3 \\
Amitriptyline & 1 & 1.4 \\
$\quad$ Nortriptyline & 1 & 1.4 \\
Sertraline & 1 & 1.4 \\
Fluoxetine & 1 & 1.4 \\
Did not refer & 1 & 1.4 \\
\hline
\end{tabular}


Table 3

Bivariate and multivariate analysis of sleep ideation and co-variables in depressed outpatients

\begin{tabular}{lllllll}
\hline & $\mathrm{OR}^{\mathrm{a}}$ & $\mathrm{IC}(95 \%)$ & $P$ & $\mathrm{OR}^{\mathrm{b}}$ & $\mathrm{IC}(95 \%)$ & $P$ \\
\hline $\mathrm{Age}^{\mathrm{c}}$ & 0.87 & $0.56-1.33$ & 0.52 & & & \\
$\mathrm{Sex}$ & & & & & & \\
$\quad$ Men & 1.13 & $0.66-1.91$ & 0.67 & & & \\
$\quad$ Women & 1.91 & $0.64-2.11$ & 0.07 & & & \\
$\begin{array}{l}\text { Duration of } \\
\quad \text { depression }\end{array}$ & 1.73 & $0.88-3.33$ & 0.11 & & & \\
$\quad$ Insomnia & 3.48 & $1.61-4.92$ & $<0.001$ & 3.1 & $1.81-4.22<0.001$ \\
$\begin{array}{l}\text { Excessive } \\
\quad 1.52\end{array}$ & $1.21-1.91$ & 0.03 & & \\
$\quad$ sleepiness & & & & & & \\
\hline
\end{tabular}

${ }^{\text {a }}$ Crude odds ratio in bivariate analysis.

b Adjusted odds ratio in multiple logistic model.

${ }^{c}$ OR relative to variation for age increase of 10 years.

${ }^{\mathrm{d}}$ OR relative to variation for month increase in duration of major depression.

Analyses were performed using the EPIINFO software program, version 6.0.

\section{Results}

The study sample consisted of 70 patients, out of which $44(62.9 \%)$ were women and $26(37.1 \%)$ men. The average age was $40.48 \pm 12.54$, and the duration in months of the depressive disorder was $6.14 \pm 1.90$.

The SSI total score of the study sample was $6.12 \pm$ 2.67. The highest SSI component scores were active suicidal ideation $(1.61 \pm 0.39)$ and specific plans for suicide $(1.51 \pm 0.40)$, followed by previous suicide attempts $(1.28 \pm 0.51)$ and passive suicidal ideation $(1.17 \pm$ $0.48)$. In the current study, $49(70 \%)$ patients were classified as having insomnia and $21(30 \%)$ as having excessive sleepiness.

The SSI scores were significantly associated with the SHQ variables regarding insomnia $\left(\chi^{2}=3.52, d f=20\right.$; $P<0,0001)$. However, no significant associations were observed with different age groups $\left(\chi^{2}=4.87, d f=69\right.$; $P=0.11)$ and with the SHQ variables regarding excessive sleepiness $\left(\chi^{2}=5.84, d f=20, P<0.11\right)$.

Insomniac patients obtained significantly higher SSI scores $(7.39 \pm 2.84)$ in relation to patients with excessive sleepiness $(3.86 \pm 1.73)(t=2.43, d f=20, P<0.05)$. Moreover, insomniac patients had significantly higher scores on the following components: active suicidal ideation, specific plans for suicide, and previous suicide attempts (Table 1).

In relation to medication intake, 27 (38.4\%) patients used antidepressants and/or hypnotics, and 43 (61.6\%) had been free of antidepressant and hypnotic medication for the past 15 days or more. Data concerning the total number of patients under antidepressant and hypnotic medication in both groups of the study sample and types of medication used are shown in Table 2. Table 3 addresses the results of bivariate and multivariate analysis. After adjustment to the confounding variable of antidepressant/hypnotic intake, the results indicated that only insomnia was significantly associated with suicidal ideation $(\mathrm{OR}=3.1,95 \% \mathrm{CI}=1.8-4.2, P<0.001)$. Suicidal ideation was not significantly associated with different age categories, sex, duration of major depressive disorder in months, or excessive sleepiness in the final logistic model.

\section{Discussion}

The main finding of this study was that suicidal ideation and sleep disorders, particularly insomnia, in patients with major depressive disorder can be associated. In the current study, insomniac patients obtained higher SSI global scores in comparison to patients with excessive somnolence. Furthermore, after adjustment for the confounding variable of medication (antidepressant and hypnotic) intake, only insomnia was significantly associated with suicidal ideation. In a previous work, insomnia based on specific items of the Schedule for Affective Disorders and Schizophrenia was considered as an important clinical symptom directly associated with suicide during the following year (Fawcett et al., 1990). The coexistence of a greater propensity for suicidal ideation among insomniac patients has similarly been reported in previous epidemiological studies (Duggan et al., 1992; Beautrais et al., 1996), in which insomnia was an important risk factor for the development both of suicidal ideation and of suicidal planning. Breslau et al. (1996) encountered a significantly increased risk for major depression and suicidality in people with prior insomnia. The excessive somnolence-major depression-suicidality association was low in the study. Excessive somnolence is a heterogeneous category, which covers specific pathological states as well as situationally determined conditions. Therefore, the heterogeneous nature of excessive somnolence may account for its relatively low association with suicidal ideation (Lichstein et al., 1994; Chellappa and Araújo, 2006). Differences regarding specific traits of suicidal ideation, such as, active and passive suicidal ideation, specific plans for suicide, and previous suicide attempts in patients with sleep disorders and depression, however, have not been fully explored (Mann et al., 1999). In the study sample, active suicide desire and specific plans for suicide components had comparatively higher SSI scores in relation to passive suicide ideation and previous suicide attempt components. According to a 
theoretical clinical model of suicidal behavior in psychiatric patients, suicidal ideation, suicide planning, insomnia, and hopelessness appear to be unique among the list of depressive symptoms, and are closely related to severe depression (Mann et al., 1999). Moreover, greater impulsitivity can partly underlie a generalized propensity to suicidal and aggressive acts. The causality between sleep, sleep disturbances and suicidality in major depression is not yet clear. Nevertheless, there is some evidence that the activity of three neurobiological systems plays a key role in the pathophysiology of suicidal behavior. This includes hyperactivity of the hypothalamus-pituitary-adrenal axis, dysfunction of the serotonergic system, and excessive activity of the noradrenergic system. While the first and the last system appear to be involved in the response to stressful events, dysfunction of the serotonergic system is thought to be trait-dependent and associated with disturbances in the regulation of anxiety, impulsivity, and hopelessness (Mann, 1998; Agargun et al., 1997b), and with alterations in the sleep-wake cycle, such as insomnia (Linnoila and Virkunnen, 1992; Riemann et al., 2001). It can therefore be hypothesized that neurobiological dysfunctions mediate the occurrence of suicidal behavior through the disturbed modulation of basic neuropsychological functions. The main clinical implication of the suicidal ideation/sleep disorders relationship is that sleep assessment can contribute to the evaluation of suicidal risk in patients with major depressive disorder.

Regarding the study limitations, the small size of the study sample $(n=70)$ restricts definite and generalized conclusions about the suicidal ideation/sleep disorders association in depressed outpatients. Moreover, the crosssectional design of this study does not permit the establishment of a cause-and-effect relationship. The use of antidepressant/hypnotic medication by some of the patients should equally be stressed. Many antidepressants and benzodiazepines can exhibit a variety of effects on the sleep architecture, such as, poor sleep perceptions, selective REM sleep deprivation and/or excessive daytime sleepiness. It is also known that these medications can result in an increase in stage 2 non-rapid eye movement (NREM) sleep, a decrease in slow-wave sleep, an increase in latency for REM sleep, a decrease in the density of rapid eye movements in REM sleep, and potential alterations in REM sleep percentage (Bernert et al., 2005). Further clinical studies with larger samples, free of antidepressant and hypnotic medication, and with a prospective design are required to assess how both insomnia and excessive sleepiness relate to suicidal ideation in patients with major depression.

In summary, sleep disorders, such as insomnia, can be associated with clinically significant suicidal ideation and specifically with active suicidal ideation and with specific plans for suicide in outpatients with depressive disorder. Therefore, sleep disturbances should be considered in the clinical assessment of depressed patients with regard to suicidal risk.

\section{References}

Agargun, M.Y., Cartwright, R., 2003. REM sleep, dream variables and suicidality in depressed patients. Psychiatry Research 119, 33-39.

Agargun, M.Y., Kara, H., Solmaz, M., 1997a. Sleep disturbance and suicidal behavior in patients with major depression. Journal of Clinical Psychiatry 58 (6), 249-251.

Agargun, M.Y., Kara, H., Solmaz, M., 1997b. Subjective sleep quality and suicidality in patients with major depression. Journal of Psychiatry 58, 377-381.

Agargun, M.Y., Cilli, A.S., Kara, H., Tarhan, N., Kincir, F., Oz, H., 1998. Repetitive and frightening dreams and suicidal behavior in patients with major depression. Comprehensive Psychiatry 39 (4), 198-202.

American Academy of Sleep Medicine, 2001. International Classification of Sleep Disorders, Revised: Diagnostic and Coding Manual. Author, Chicago.

American Psychiatric Association, 1994. Diagnostic and Statistical Manual of Mental Disorders (DSM-IV), 4th ed. American Psychiatric Press, Washington, DC.

Andrade, M.M.M., Benedito-Silva, A.A., Menna-Barreto, L., 1992. Correlates between morning-eveningness character and sleep habits. Brazilian Journal of Medical and Biological Research 25, 835-839.

Beautrais, A.L., Joyce, P.R., Mulder, R.T., Fergusson, D.M., Deavoll, B.J., Nightingale, S.K., 1996. Prevalence and comorbidity of mental disorders in persons making serious attempts: a casecontrol study. American Journal of Psychiatry 153, 1009-1014.

Beck, A.T., Kovacs, M., Weissmann, A., 1979. Assessment of suicidal intention: the Scale for Suicidal Ideation. Journal of Consultant Clinical Psychology 47, 342-352.

Bernert, R.A., Joiner Jr., T.E., Cukrowicz, K.C., Schmidt, N.B., Krakow, B., 2005. Suicidality and sleep disturbances. Sleep 28 (9), $1135-1141$

Bertolote, J.M., Fleishmann, A., 2002. A global perspective in the epidemiology of suicide. Suicidology 7 (2), 6-7.

Breslau, N., Roth, T., Rosenthal, L., 1996. Sleep disturbance and psychiatric disorders: a longitudinal epidemiological study of young adults. Biological Psychiatry 39, 411-418.

Chellappa, S.L., Araújo, J.F., 2006. Excessive daytime sleepiness in patients with depressive disorder. Revista Brasileira de Psiquiatria $28(2), 1-7$.

Cunha, J., 2001. Manual da versão em português das Escalas de Beck. Casa do Psicólogo, São Paulo.

Duggan, C.F., Sham, P., Lee, A.S., Murray, R.M., 1992. Can future suicidal behavior in depressed patients be predicted? Journal of Affective Disorder 22, 111-118.

Fawcett, J., Scheftner, W.A., Fogg, L., Clarck, D.C., Young, M.A., 1990. Time-related predictors of suicide in major affective disorder. American Journal of Psychiatry 147, 1189-1194.

Landis, J.R., Koch, G.G., 1977. The measurement of observer agreement for categorical data. Biometrics 33 (1), 159-174.

Lichstein, K.L., Wilson, N.M., Noe, S.L., 1994. Daytime sleepiness in insomnia: behavioral, biological indices. Sleep 17, 693-702.

Linnoila, V.M., Virkunnen, M., 1992. Aggression, depression and serotonin. Journal of Clinical Psychiatry 53, 46-51. 
Mann, J.J., 1998. The neurobiology of suicide. Nature Medicine 4, $25-30$.

Mann, J.J., Waternaux, C., Haas, G.L., Malone, K.M., 1999. Towards a clinical model of suicidal behavior in psychiatric patients. American Journal of Psychiatry 156, 181-189.

Ohayon, M.M., 2000. Epidemiology of insomnia: what we know and what we still need to learn. Sleep Medicine Reviews 6 (2), 97-111.

Riemann, D., Berger, M., Voderholzer, U., 2001. Sleep and depressionresults from psychobiological studies: an overview. Biological Psychology 57, 67-103.
Sabo, E., Reynolds, C.F., Kupfer, D.J., Bergman, S.R., 1991. Sleep, depression and suicide. Psychiatry Research 36, 265-277.

Schmidtke, A., Bille-Brahe, U., De Leo, D., Kerkhof, A., Wasserman, D., 2004. Suicidal Behavior in Europe: Results from the WHO/EURO Multicentre Study on Suicidal Behavior. Hogrefe and Huber, Göttingen.

Singareddy, R.K., Balon, R., 2001. Sleep and suicide in psychiatric patients. Annals of Clinical Psychiatry 13, 93-101.

Tankensen, A., Tuomilehto, J., Viinamaki, H., Vartiainen, E., Lehtonen, J., Puska, P., 2001. Nightmares as predictors of suicide. Sleep 24 (7), 844-847. 